\title{
Amine-Ligated Approach for the Synthesis of Extra-Large-Pore Zinc Phosphites with qtz-h and bnn Topologies
}

\author{
Kangcai Wang, ${ }^{\ddagger}$ Yixuan Bian, ${ }^{\star}$ Jing Li, Dingguo $\mathrm{Xu},{ }^{*}$ and Zhien Lin* \\ College of Chemistry, Sichuan University, Chengdu 610064, People's Republic of \\ China \\ \$ These authors contributed equally. \\ * To whom correspondence should be addressed. Tel: +86-28-85412284. E-mail: \\ dgxu@scu.edu.cn (D. Xu); zhienlin@scu.edu.cn (Z. Lin)
}




\section{Physical measurements:}

IR spectra ( $\mathrm{KBr}$ pellets) were recorded on a Nicolet Impact 410 FTIR spectrometer. Powder X-ray diffraction (XRD) data were obtained using a Shimadzu XRD-6100 diffractometer with $\mathrm{Cu}-\mathrm{K} \alpha$ radiation $(\lambda=1.5418 \AA)$. The thermogravimetric analyses were performed on a Netzsch STA 449c analyzer in a flow of $\mathrm{N}_{2}$ with a heating rate of $10{ }^{\circ} \mathrm{C} / \mathrm{min} . \mathrm{N}_{2}$ and $\mathrm{H}_{2}$ gas sorption measurements were performed on a Micromeritics ASAP 2020 surface area and pore size analyzer. The photoluminescent spectra were measured on a Perkin-Elmer LS 55 luminescence spectrometer equipped with a $450 \mathrm{~W}$ xenon lamp. Powder second-harmonic generation ( $\mathrm{SHG}$ ) signals were measured by means of the Kurtz and Perry method. ${ }^{1}$ The fundamental wavelength was $1064 \mathrm{~nm}$ and was generated by a Q-switched Nd:YAG laser. The SHG wavelength was $532 \mathrm{~nm}$. KDP

powder was used as a reference. Single crystal X-ray diffraction data were collected on a New Gemini, Dual, $\mathrm{Cu}$ at zero, EosS2 diffractometer at room temperature. The crystal structures were solved by direct methods. The structures were refined on $F^{2}$ by full-matrix least-squares methods using the SHELXTL program package. ${ }^{2}$

\section{Reference}

(1) S. K. Kurtz, T. T. Perry, J. Appl. Phys. 1968, 39, 3798.

(2) G. M. Sheldrick, Acta Cryst., Sect. A 2008, 64, 112. 


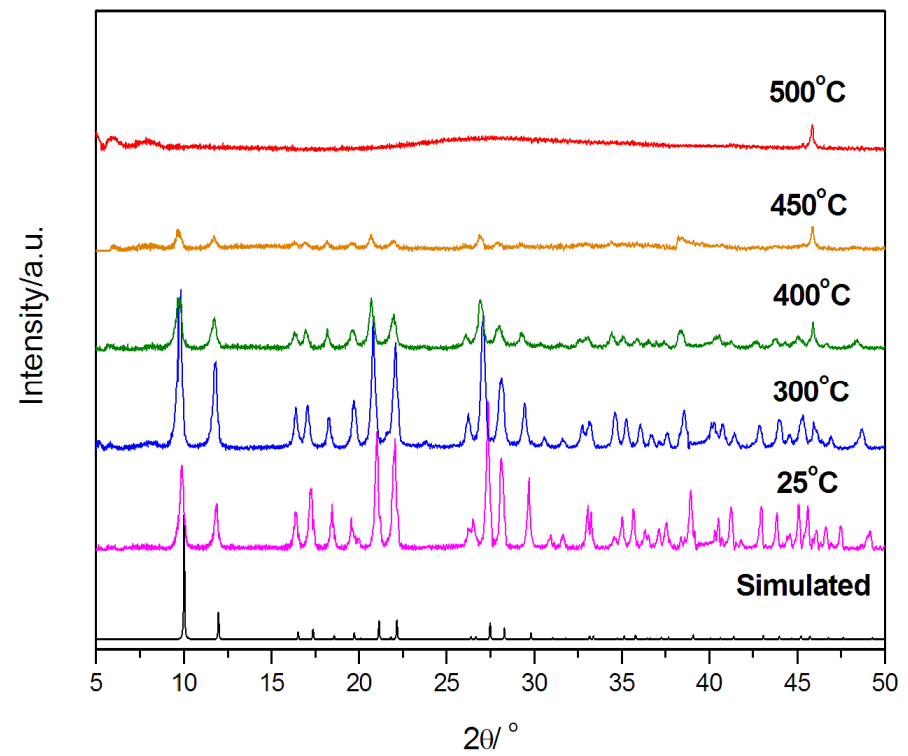

Figure S1. Simulate powder XRD pattern of SCU-18 and the as-synthesized sample upon treatment at different temperature.

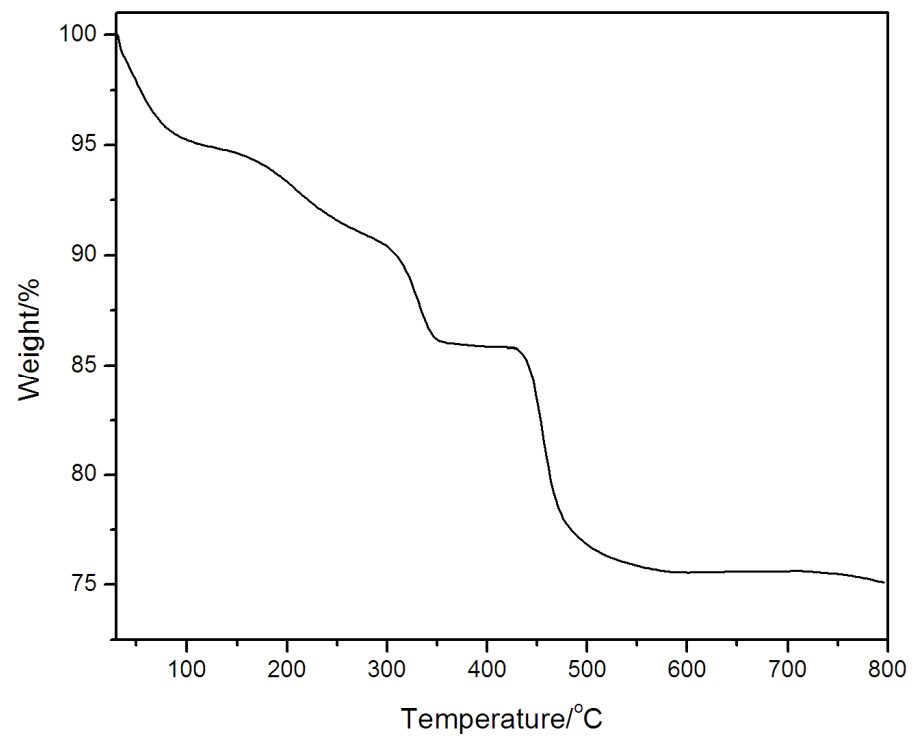

Figure S2. TGA curve of SCU-18. 


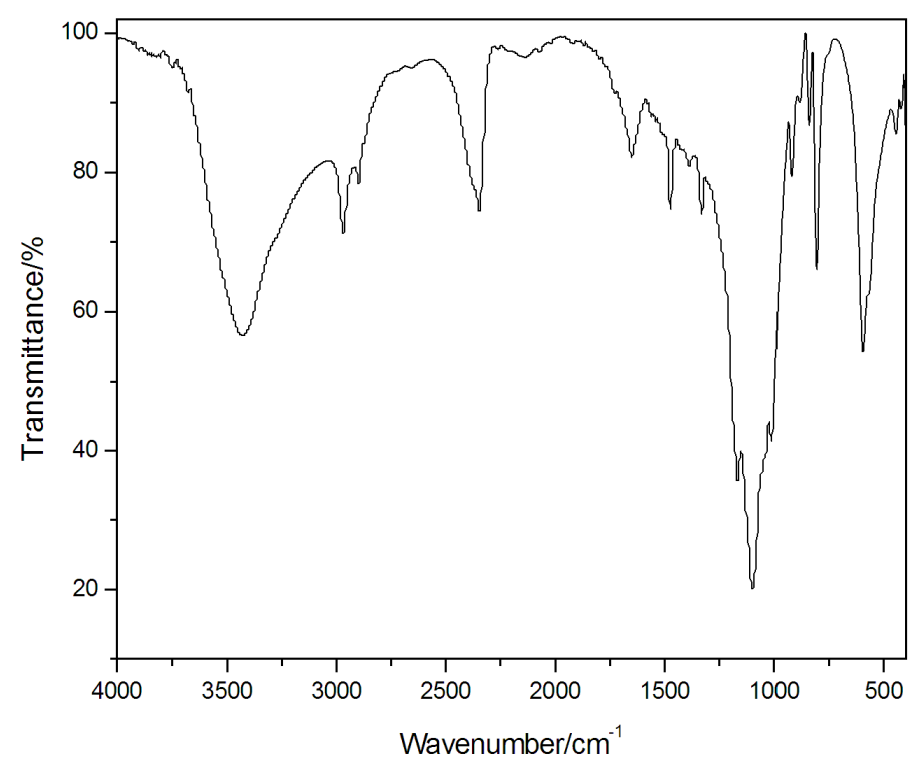

Figure S3. IR spectrum of SCU-18.

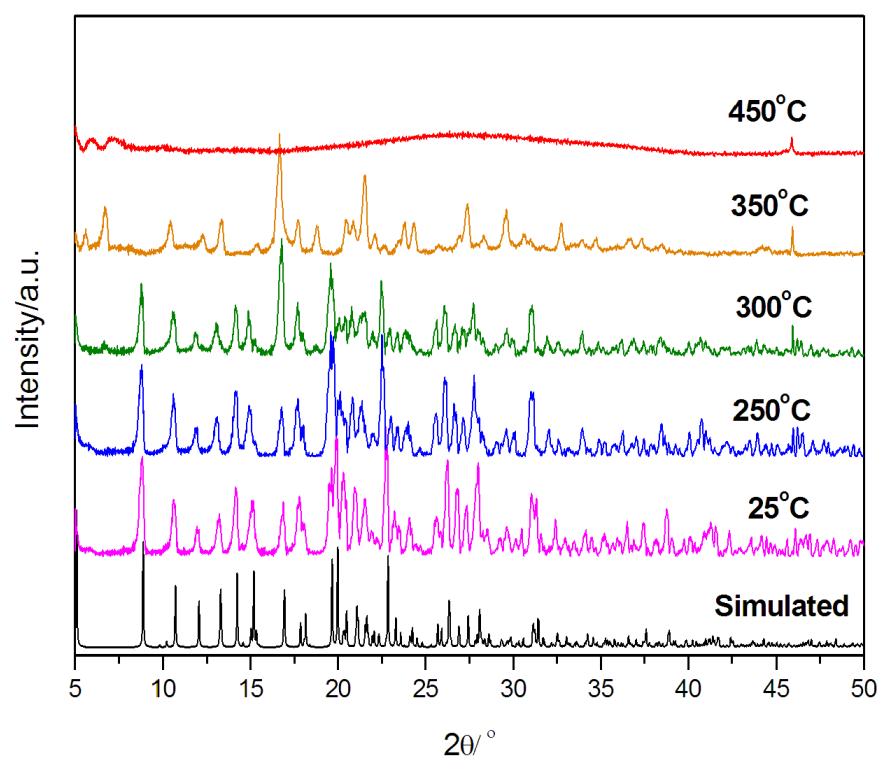

Figure S4. Simulate powder XRD pattern of SCU-20 and the as-synthesized sample upon treatment at different temperature. 


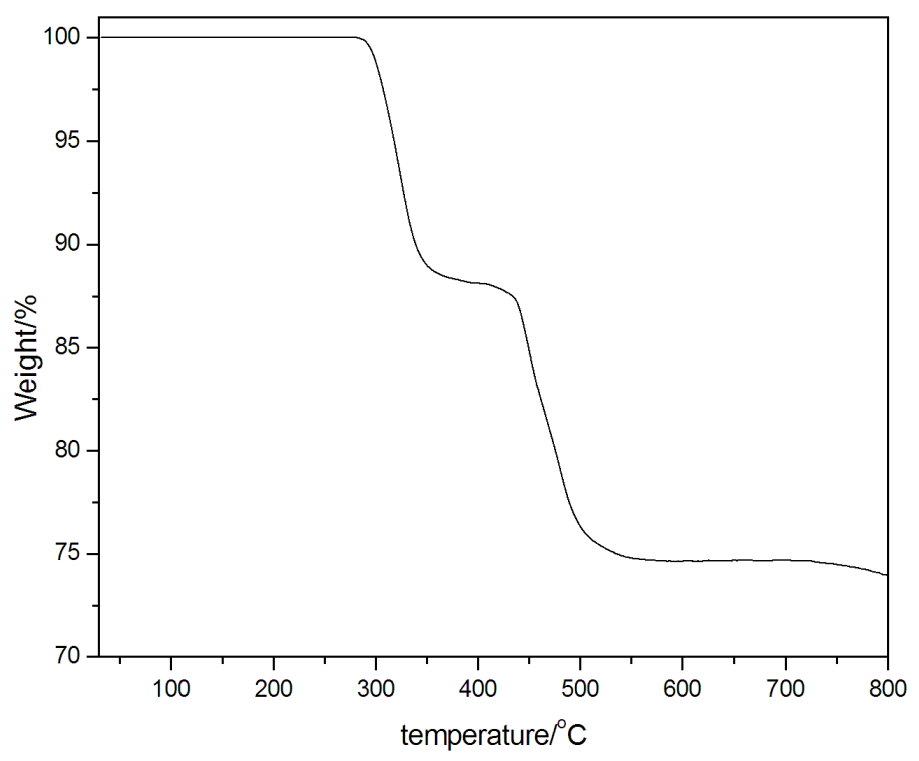

Figure S5. TGA curve of SCU-20.

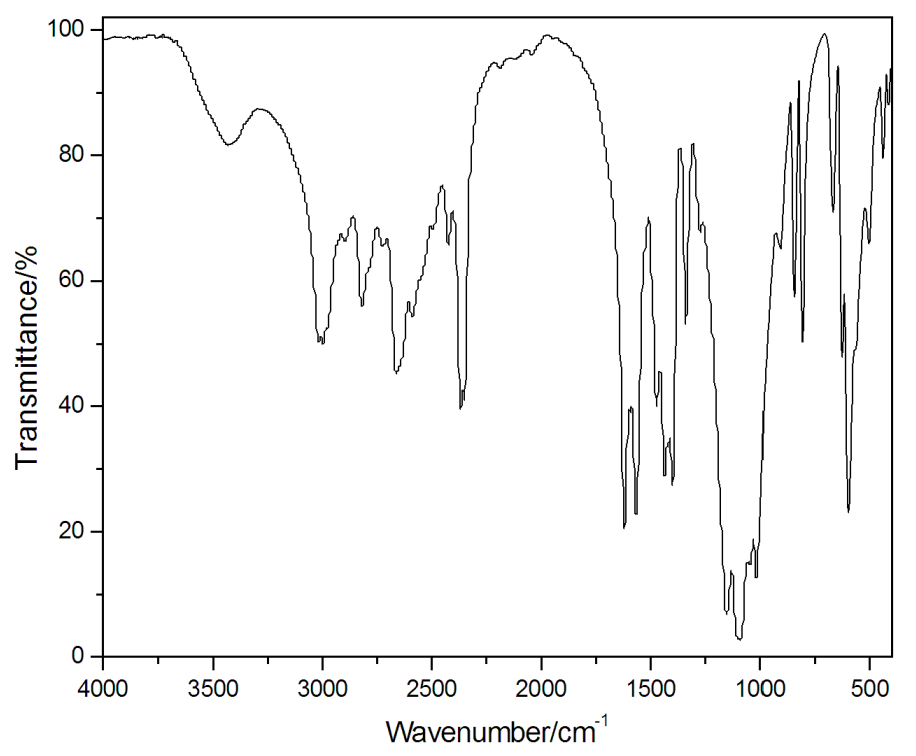

Figure S6. IR spectrum of SCU-20. 


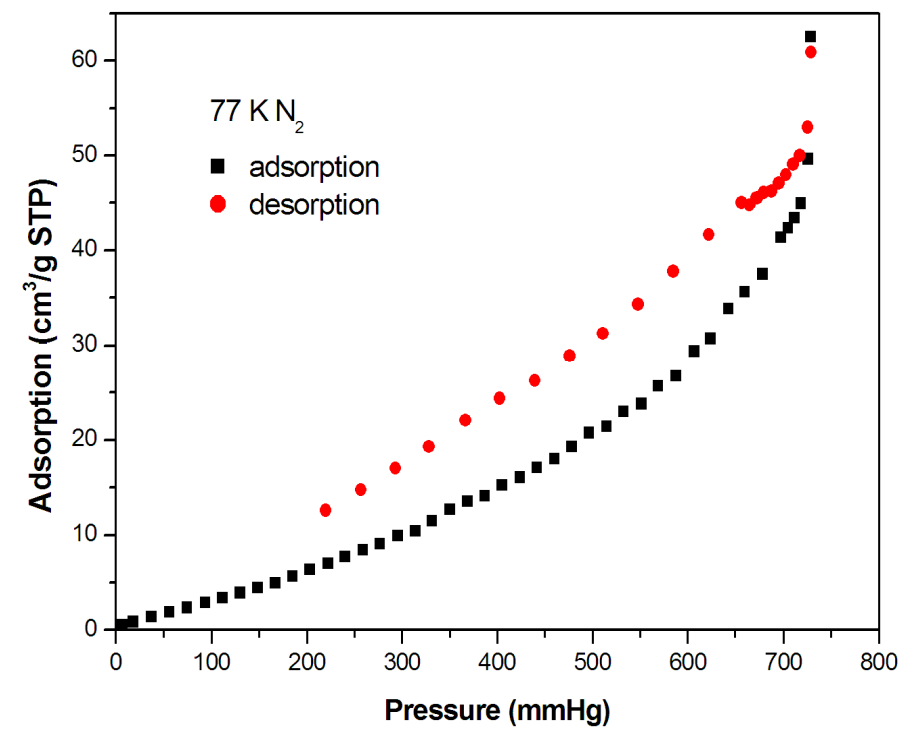

Figure S7. The $\mathrm{N}_{2}$ adsorption-desorption isotherm of SCU-18 at $77 \mathrm{~K}$.

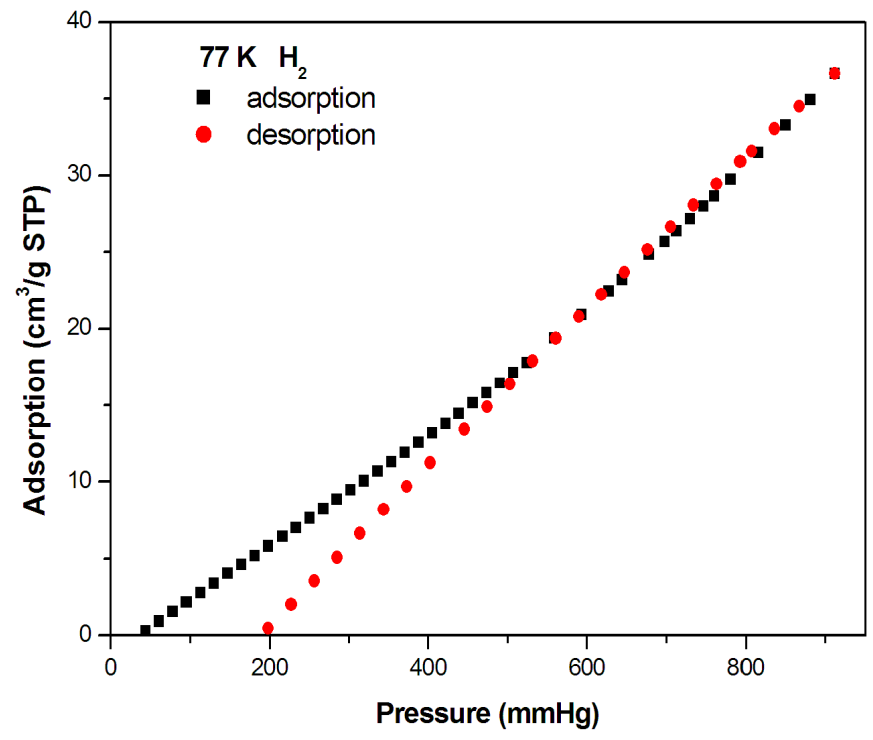

Figure S8. The $\mathrm{H}_{2}$ adsorption-desorption isotherm of SCU-18 at $77 \mathrm{~K}$. 


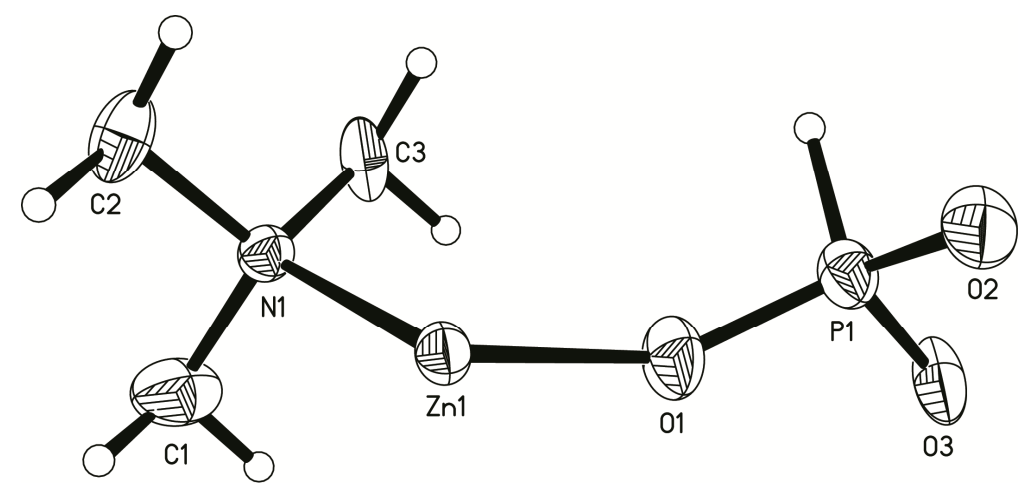

Figure S9. ORTEP plot of the asymmetric unit of SCU-18, showing the labeling scheme and the $30 \%$ probability displacement ellipsoid.

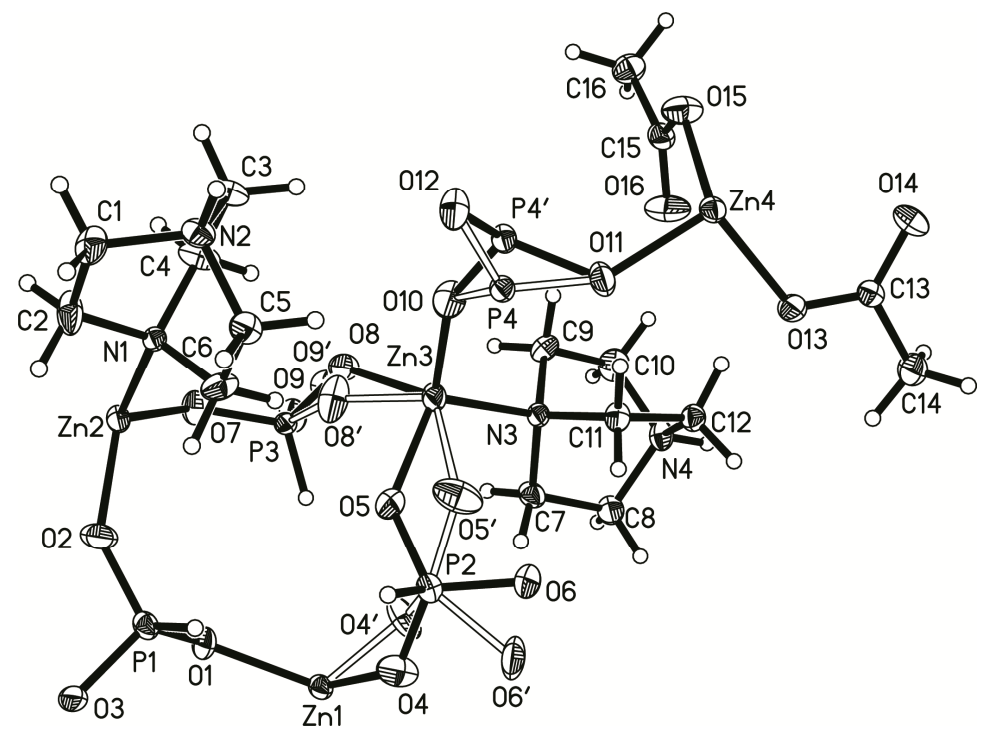

Figure S10. ORTEP plot of the asymmetric unit of SCU-20, showing the labeling scheme and the $30 \%$ probability displacement ellipsoid. 